\title{
Molecular genetics: science and technology regulation
}

\author{
Rajalakshmi Swaminathan and Ajay Parida*
}

\begin{abstract}
The 23rd Annual Dialogue series at the MS Swaminathan Research Foundation aimed at suggesting important inputs in the regulatory process for the approval and commercialisation of genetically modified crops.
\end{abstract}

Keywords: genetically modified technologies, regulatory process, public acceptance

\section{Opinion}

A brainstorming and engaging dialogue on the merits of genetically modified (GM) crops to augment the agrarian crisis was held in Chennai, India, by the MS Swaminathan Research Foundation to commemorate 60 years since the discovery of the DNA double helix structure. The Foundation brought together all stakeholders in the agriculture sector, including molecular biologists, plant breeders, farmers' associations and civil society representatives from both national and international institutions, to help produce definite guidelines on the Regulatory Authority Bill that is before the Indian Parliament for deliberations and discussions.

The three-day consultation was attended by 60 delegates from premier institutions, industry, policymakers and the civil society network. Participants felt the need for dialogue and for clarity in understanding the concerns and challenges in the commercialisation of GM crops. Prof MS Swaminathan, Emeritus Chairman of the MS Swaminathan Research Foundation, reiterated in his inaugural address that 'the bottom line of our national agricultural biotechnology policy should be the economic well-being of farm families, food security of the nation, health security of the consumer, biosecurity of agriculture and health, protection of the environment and the security of national and international trade in farm commodities'. Nine technical sessions on various aspects of food, health, nutrition security, examples of how research leads to product development, recent technological advances in molecular biology, industry

\footnotetext{
* Correspondence: ajay-ed@mssrf.res.in

MS Swaminathan Research Foundation, 3rd Cross Street, Taramani Institutional Area, Chennai 600 113, India
}

perspectives of research, key regulatory concerns and processes were discussed and debated at length.

A few success stories were presented from premier publicly funded institutions and from industry, namely Nirmal Seeds, Metahelix and Avesthagen Ltd. Delegates felt the need to create an appropriate infrastructure that would nurture innovation and stimulate fundamental research in biotechnology and thus bridge the gap between the scientific community and industry. Premier institutions were represented by senior scientists from the Central Rice Research Institute (CRRI), the Indian Agricultural Research Institute (IARI), the International Livestock Research Institute (ILRI), the National Resource Centre on Plant Biotechnology (NRCPB), the Indian Council for Agricultural Research (ICAR), the International Crops Research Institute for Semi Arid Tropics (ICRISAT), the National Institute of Plant Genome Research (NIPGR), and Syngenta Foundation. Civil society groups like Greenpeace, Jatan Trust, Thanal and the Council for Responsible Genetics were active participants in the dialogue. Senior members from the Government of India included the Secretary for the Department of Biotechnology, the Additional Secretary for the Ministry of the Environment and Forests and the Chairman of the Genetic Engineering Approval Committee (GEAC). International delegates representing the South African and UK governments, and the Norman Borlaug Institute gave insights into the regulatory mechanisms and public acceptance in their countries.

This consultation saw participants share very healthy and constructive suggestions for incorporation into the new Biotechnology Regulatory Bill that is under active consideration by the Indian Parliament. Participants also 
felt that products needs to be prioritised to give the optimal use of the available resources. This would set the roadmap for policy development and the commercialisation of GM crops. Seven key recommendations emerged after three days of deliberations:

1. The Department of Medical Research and ICMR should establish a medical technology assessment board to verify claims and grant approval only to those technologies that can advance the cause of human health.

2. A coordinated project, organised in a mission mode, is necessary so that scientific advances in bioremediation can be used for the treatment of polluted industrial wastewater to provide safe potable water and for cleaning soils contaminated with heavy metals.

3. In agricultural and food biotechnology, a welldesigned needs assessment should be introduced to facilitate decisions on publicly funded genetic modification research.

4. The regulatory body needs to base its decisions on the precautionary principle, using the Cartagena Protocol on Biosafety, which is the internationally approved protocol.

5. Genetic literacy at the grassroots panchayat level and giving school and college students a better understanding of the advances in science will help the Government of India to disseminate information about GM crops across all strata of society. A media resource centre that will demystify and publish information regarding GM crops will further help in the broad understanding of the subject. Training on issues related to intellectual property, regulatory affairs and commercialisation of GM crops needs to be undertaken by accredited institutions.

6. The use of genetic advances for public good needs to be funded by the government and by publicly funded institutions to increase acceptance of the technology and its transfer to the field. Such institutions will naturally ensure social inclusion and equal access to the technology.

7. It was felt that the hidden hunger and the micronutrient deficiency in the diet can be largely eliminated by integrating nutritionally rich crops and plants and promoting nutri-farms or homestead gardens in every home across rural India.

Most participants felt that while there is adequate expertise and facilities available in the country, additional accredited laboratories dedicated to evaluating and testing the biosafety of GM crops need to be established. The agronomic parameters for each crop need to be clearly chalked out for a precise evaluation. Regular meetings of the approval and regulatory committees will accelerate the assessment and evaluation of the crops that are positioned for approvals. Participants stressed the need for greater transparency of the evaluation and assessments conducted by the regulatory authority.

Delegates agreed on the need for more public-private partnerships and more collaboration within India and with international institutions to harness the potential of biotechnology to improve the quality of life, particularly in developing countries. To summarise, participants, while acknowledging the existence of a fairly robust regulatory system in the country, expressed the need to create an independent autonomous regulatory authority that would bring together all the expertise. The coordination of the various stakeholders would then occur in one agency, which could evaluate and strengthen the regulatory processes more efficiently and in a time-bound manner, before commercialisation of GM products. Setting up these systems and independent testing laboratories of repute, will ensure scientific rigour and thereby result in greater public acceptance of GM technologies.

\section{Competing interests}

The authors declare that they have no competing interests.

\section{Authors' contributions}

SR wrote the manuscript and ensured that all viewpoints deliberated during the sessions were noted and incorporated. AP gave critical inputs in the draft recommendations that formed the basis for the discussions. Both authors read and approved the final manuscript.

\section{Acknowledgements}

This dialogue was supported by ICRISAT and Harvest Plus and it was organised by the MS Swaminathan Research Foundation. The inputs and suggestions from Prof MS Swaminathan and all delegates are acknowledged.

Received: 5 July 2013 Accepted: 16 April 2014

Published: 12 May 2014

doi:10.1186/2048-7010-3-10

Cite this article as: Swaminathan and Parida: Molecular genetics: science and technology regulation. Agriculture \& Food Security 2014 3:10. 